\title{
0 conceito de (re)tradução sob uma abordagem processual: um estudo empírico-experimental baseado em rastreamento ocular ${ }^{1}$ The concept of (re)translation from a process-based approach: An empirical- experimental study based on eye-tracking
}

\author{
Gleiton Malta*
}

\begin{abstract}
RESUMO: Desde o surgimento da hipótese da retradução no início da década de 1990 , vários teóricos vêm se debruçando sobre o tema. A maioria dos estudos tem como objetivo principal averiguar se os textos traduzidos mais recentemente possuem características que os tornem menos assimiladores que os textos traduzidos há mais tempo, em relação ao mesmo texto original. Contudo, tanto as pesquisas como o próprio conceito de retradução são baseados no produto tradutório. Este artigo apresenta o conceito de (re)tradução sob uma abordagem processual. Por tanto, lançou mão de um estudo empírico-experimental no qual catorze participantes, todos com alguma experiência em tradução, tiveram seu processo capturado em tempo real por equipamento de rastreamento ocular. Como resultado, propõem-se, além das definições de (re)tradução, como tarefa e como processo, um modelo do processo de (re)tradução baseado nos dados de rastreamento ocular. (Re)visitar a retradução com os olhos do processo possibilitou a extração e análise de dados ainda não explorados pela literatura e que, futuramente, poderão ser utilizados em novas pesquisas processuais, tanto na rama pura como na aplicada do campo disciplinar dos Estudos da Tradução, mais especificamente, pela didática da tradução.
\end{abstract}

PALAVRAS-CHAVE: (Re)tradução. Abordagem processual. Modelagem. Estudo experimental. Rastreamento Ocular.

\begin{abstract}
Since the dawn of the (re)translation hypothesis in the early 1990s, several theorists have been investigating the subject. Most studies have the aim of finding out if the most recently translated texts have characteristics that make them less absorbing than the translated texts from some time ago. However, both studies and the very concept of retranslation are based on the translational product. This article introduces the concept of (re)translation from a process-based approach. Therefore, it deploys an empiricalexperimental study in which fourteen participants, all with some experience in translation, had their process captured in real time by an eye-tracking equipment. As a result, it proposes - in addition to the definitions of (re)translation as a task and as a process - a process model of (re)translation based on eye-tracking data. (Re)visiting retranslation with the eyes of the process made the extraction and data analysis possible. These data may be used in the future in the applied branch of Translation Studies field, especially the teaching of translation.
\end{abstract}

KEYWORDS: (Re)translation. Processbased Approach. Modeling. Experimental Study. Eye-tracking.

\footnotetext{
${ }^{1}$ Este artigo apresenta parte da pesquisa realizada pelo autor em seu doutorado, realizado na Faculdade de Letras da Universidade Federal de Minas Gerais, com orientação da prof. Dra. Adriana Pagano e coorientação do prof. Dr. Igor A. L da Silva.

* Departamento de Línguas Estrangeiras e Tradução (LET)/ Instituto de Letras (IL)/Universidade de Brasília UnB.
} 


\section{Introdução}

Segundo a Routlegde Encyclopedia of translation studies (BAKER; MALMKJAER, 1998), o termo retradução, embora algumas vezes seja utilizado para se referir à tradução indireta, é mais comumente empregado para definir o ato de traduzir um trabalho que já foi previamente traduzido de uma dada língua-fonte para uma mesma língua-alvo (TAHIRGÜRÇALAR, 1998, p. 233). Ainda que seja uma prática bastante antiga, é a partir do surgimento da "hipótese da retradução" (doravante HR) apresentada por Berman (1990) que o tema passa a ser analisado com maior sistematicidade e rigor.

Este artigo traz o resultado de parte de uma pesquisa em nível de doutorado realizada no âmbito do Laboratório Experimental de Tradução (LETRA) da Faculdade de Letras (FALE) da Universidade Federal de Minas gerais (UFMG), em parceria com o Departamento de Línguas Estrangeiras e Tradução (LET) da Universidade de Brasília (UnB), na qual a retradução é explorada por meio de uma abordagem processual. Trata-se de um estudo exploratório, empírico-experimental, vinculado ao campo disciplinar dos Estudos da Tradução, mais especificamente aos estudos descritivos orientados ao processo.

Dentro da abordagem processual dos Estudos da Tradução, desenhos experimentais, os quais contemplam diferentes tipos de tarefas ${ }^{2}$ com diferentes objetivos de pesquisa, são propostas de estudos empíricos capazes de trazer à tona o que está subjacente ao ato tradutório, e assim, por meio da inferência, acessarem indiretamente o processo de tradução. Nesse sentido, tarefas envolvendo tradução, pós-edição e revisão, estão entre os tipos de tarefas mais explorados pelos pesquisadores.

O objetivo neste artigo é: i) apresentar um modelo processual da (re)tradução a partir de dados de rastreamento ocular, além de ii) apresentar duas novas definições de (re)tradução, ou seja, como processo e como tarefa. Tanto o modelo como as definições foram elaborados a partir de dados de rastreamento ocular, extraídos em tempo real.

À guisa de esclarecimento, o termo (re)tradução é usado para referir-se ao processo, em contraste com o termo já cunhado, retradução (retranslation), utilizado para referir-se ao produto. Essa diferenciação se faz necessária devido às especificidades de cada uma das abordagens, ou seja, processo e produto. No primeiro caso, utiliza-se o termo com os parênteses,

\footnotetext{
${ }^{2}$ Entende-se por tarefa a atividade a ser realizada pelo participante de pesquisa para a coleta de dados em tempo real. Nos Estudos processuais da tradução é comum o uso de tarefas em desenhos experimentais, pelos quais os dados do processo são coletados.
} 
(re)tradução, pois defendemos que dentro desse processo há, explícito, um processo eminente de tradução, como será mostrado na seção 4 dos Resultados. No segundo caso, como o objeto de estudo é estático, ou seja, o texto, é possível a realização de uma análise estritamente voltada para o fenômeno da retradução, sem, necessariamente, referir-se à tradução em si, ainda que essa também esteja presente.

Uma pesquisa dessa natureza é relevante para o campo disciplinar dos Estudos da Tradução, pois apresenta dados processuais inéditos de uma tarefa ainda não explorada pelos estudos com abordagem processual. Além disso, contribui com as definições de (re)tradução, como processo e como tarefa, que poderão ser úteis em novas pesquisas, envolvendo tanto o tema, quanto a abordagem. Agrega-se a isso o fato de que retraduzir é uma atividade frequente, tanto dentro e fora do Brasil (SUSAN-SARAJEVA, 2003; PALOPOSKI; KOSKINEN, 2010; MALTA; RAEL; 2015).

Este artigo está organizado em seções, incluindo esta Introdução. Na seção 2, dispõese a fundamentação teórica em duas subseções, a primeira com ênfase nos estudos sobre retradução, e a segunda nos estudos da tradução com abordagem processual com base em pesquisa experimental. A metodologia de pesquisa é o tema da seção 3, cujo detalhamento se centrará na metodologia de coleta e análise dos dados. Na seção 4, discutem-se os dados e apresentam-se o modelo processual de (re)tradução e suas definições como processo e como tarefa. Na seção 5, são feitas as considerações finais do estudo, incluindo suas limitações e sugestões para futuras pesquisas.

\section{Pressupostos teóricos: da retradução às pesquisas voltadas para o processo tradutório.}

\subsection{Retradução}

Se do ponto de vista processual há carência de estudos que tenham a retradução como tarefa principal de investigação, no âmbito dos estudos do produto tradutório há uma série de trabalhos desenvolvidos (e.g., SUSAN-SRAJEVA, 2003; PALOPOSTKI; KOSKINEN, 2001, 2010; DASTJERDI; MOHAMMADI, 2013; MALTA; RAEL, 2015). Como elencado na Introdução deste artigo, apesar de bastante antigo, o tema ganhou status de objeto de pesquisa a partir do texto basilar de Berman (1990), La retraduction comme espace de la traduction, publicado em um número da revista Palimpsestes (cf. BENSIMON, 1990). Para Berman (1990), ao passo que as traduções "envelhecem", os textos originais continuam eternamente “jovens” (BERMAN, 1990, p. 1). Isso ocorreria devido ao contexto histórico, social, cultural, 
entre outros motivos, em que as traduções estão inseridas. Para Berman (1990), toda tradução realizada após a primeira tradução de uma obra é uma retradução. Dessa forma, segundo a HR, as traduções mais recentes se aproximam mais do texto-fonte que as mais antigas, ou seja, enquanto as primeiras tenderiam a ser mais "domesticadoras" (VENUTTI, 1995), as mais recentes tenderiam a ser mais "estrangeirizadoras" (idem).

Gambier (1994), por outra parte, define a retradução como "uma nova tradução, numa mesma língua, de um texto já traduzido, em totalidade ou em parte"33 (GAMBIER, 1994, p. 413). Tanto Berman como Gambier convergem no sentido de que vários motivos levam um texto a ser retraduzido. Eles vão desde a atualização de uma tradução que já não representa para o público atual a obra original, até questões comerciais, como o lançamento de novas (re)traduções em datas comemorativas. O fato é que, segundo os autores, a primeira tradução tem sempre uma tendência a ser mais assimiladora em função do argumento de se garantir sua legibilidade pelo público alvo. Nesse caso, a retradução seria, então, "um retorno ao textofonte" ${ }^{4}$ (GAMBIER, 1994, p. 414); portanto, se aproximaria mais dele que a primeira tradução. Esse seria o princípio da hipótese da retradução, ou seja, as traduções mais recentes se aproximam mais do texto-fonte, enquanto as mais antigas se distanciam.

Segundo diversos estudos, são vários os motivos pelos quais uma obra é retraduzida. Berman (1990) assevera que as traduções “envelhecem", ao passo que o texto-fonte se mantém jovem. Para Susan-Sarajeva (2003), o motivo que leva um texto e ser retraduzido está muito mais no contexto receptor que no texto em si, já que muitas vezes as retraduções se encontram separadas por períodos de tempo curtos. Para Milton (2001) e Paloposki e Koskinen (2010), o mercado editorial e a garantia nas vendas são fortes influências na hora de se optar pelas retraduções. O fato é que a ideia que subjaz à HR é a de que quanto mais se distancia cronologicamente do texto-fonte e quantas mais traduções são feitas, melhor será o produto tradutório. Essa ideia temporal, baseada na progressão histórica, foi refutada por SusanSarajeva (2003) que, como afirmado anteriormente, elenca diversos exemplos de traduções realizadas em um período curto de tempo.

Se por um lado Susan-Sarajeva refuta a HR, por outro, Dastjerdi e Mohammadi (2013), em uma pesquisa de corpus realizada em três capítulos da obra Pride and Prejudice (1813), encontram indícios que corroboram a hipótese de Berman. Baseados nesses autores, Cremonez

\footnotetext{
3 "serait una nouvelle traduction, dans une même langue, d'um texte déjà traduit, en entier ou en partie"

4 "un retour au texte-source"
} 
e Da Silva (2015), utilizando uma metodologia fundamentada na Linguística de Corpus, analisaram diferentes traduções de The picture of Dorian Gray, de Oscar Wide, e também encontraram indícios a favor da HR.

Destarte, em outra pesquisa cuja metodologia privilegia o contraste entre o texto-fonte e pelos menos duas traduções desse mesmo texto, Malta e Rael (2015) chamam a atenção para o fato de que a hipótese pode ser corroborada ou refutada em um mesmo estudo. Tendo como objeto de estudo La casa de los espíritus, de Isabel Allende e duas de suas traduções, uma datada de 1984 e a outra de 2014, os autores concluem que, estruturalmente, a primeira tradução se aproxima mais do texto-fonte que a segunda, o que refuta a HR. Nesse caso, a análise proposta foi estritamente linguística, privilegiando similitudes e diferenças, proximidades e distanciamento no que tange o uso de advérbios, numerais, entre outros. No entanto, destacam a "importância de mais estudos empíricos comparativos em tradução, tomando por base ora a semântica, ora a estilística, ora a estrutura e assim por diante" já que, em diversos momentos, uma mesma tradução pode ser mais assimiladora ou não (MALTA; RAEL, 2015, p. 95).

Em todos os estudos citados, o objeto de estudo é o produto tradutório. Este estudo foge desse paradigma e analisa a (re)tradução como processo, é, portanto, um estudo exploratório possibilitado por métodos de investigação empírico-experimentais dentro da abordagem processual da tradução. Isso significa que, por ser exploratório, não almejamos refutar ou corroborar a HR, mas somente registrar o fluxo de movimentação ocular que os participantes possuem ao se depararem com uma tarefa ainda não explorada pelos estudos processuais, cujas principais características são elencadas na seguinte subseção.

\subsection{Abordagem processual nos Estudos da Tradução}

O interesse de pesquisadores no processo de tradução data de 1980. Para Rodrigues (2002, p. 24), esse interesse em investigar o processo tradutório advém, basicamente, de dois motivos: o primeiro tem a ver com percepção de que os modelos teóricos até então vigentes "não representavam a realidade da tradução tal como vivenciada pelos próprios tradutores", além de serem elaborados, frequentemente, sem embasamento empírico. O segundo motivo, ainda segundo Rodrigues (2002, p. 24), representa a preocupação de pesquisadores com o fato de o tradutor estar, quase sempre, ausente de qualquer "fundamentação teórica tanto sobre o produto quanto sobre o processo da tradução". 
Desde então, várias tentativas têm sido empreendidas com o intuito de se capturar as diferentes facetas que envolvem o processo tradutório. Segundo Dragsted e Carl (2013), os modelos criados para representar os processos envolvidos na produção da tradução têm em comum a relação estabelecida entre um texto-fonte (insumo) e um texto-alvo (produto), bem como a interação entre outros recursos, como os conhecimentos anteriores (background knowledge), armazenados na memória de longo prazo. Para Dragsted e Carl (2013), a natureza da relação que envolve texto-fonte e o texto-alvo é descrita de diferentes formas pelas pesquisas processuais.

Em décadas anteriores, pesquisas com abordagem processual utilizavam diferentes tipos de metodologias (DRAGSTED; CARL, 2013), o que impossibilitava a comparação dos resultados advindos de dados quantitativos e qualitativos concernentes aos estudos processuais. Essa ausência de uma metodologia comum impedia não só a comparação de dados, mas também que desenhos experimentais fossem replicados com diferentes tipos de participantes e pares linguísticos, o que também resultava em falta de rigor metodológico (TIRKKONEN-CONDIT, 2000).

A inserção de novos instrumentos de coleta de dados, como os registradores de teclado e mouse e os rastreadores oculares, veio contribuir com o incremento e com o surgimento de novas metodologias de pesquisa processual em tradução, dando maior suporte aos dados advindos dos já utilizados protocolos verbais. As informações observáveis coletadas por meio de rastreadores oculares e programas de gravação de acionamentos de teclas e mouse podem ser indicativos, entre outros, de processos cognitivos não observáveis que têm lugar durante as tarefas de tradução (PAVLOVIĆ; JENSEN, 2009).

As pesquisas acerca dos movimentos oculares foram sistematizadas por Rayner (1998), que sugere uma ligação entre o movimento dos olhos e o processamento de informação. Just e Carpenter (1980) nomearam essa associação como princípio da relação olho-mente (eyemind assumption), segundo o qual operações cognitivas têm impacto nos movimentos dos olhos durante a execução de uma tarefa ${ }^{5}$. No caso deste artigo, são apresentados dados de rastreamento ocular para registrar o fluxo de acessos ao texto-fonte, às traduções e ao textoalvo, constantes do experimento, conforme detalhado mais adiante na Seção 3 dedicada à

\footnotetext{
${ }^{5}$ Mais informações sobre o assunto podem ser obtidas em Rayner (1980), Just e Carpenter (1980), Sekino (2015), Malta (2015), entre outros.
} 
metodologia, não sendo foco deste trabalho o processamento e o esforço cognitivo (cf. JUST; CARPENTER, 1980; RAYNER, 1998; MALTA, 2015; SEKINO, 2015).

Em tradução, os rastreadores oculares são utilizados para a coleta de dados referentes a variáveis como: olhar (gaze), fixação, sacada, transição, visitas, dilatação de pupila, quase sempre relacionadas ao processamento cognitivo. Com esses equipamentos, é possível mapear o olhar dos participantes e aferir onde incide maior atenção visual. Neste estudo são analisados dados concernentes a fixações, visitas e transições, cujas definições estão a seguir.

- Fixação: São períodos em que os olhos permanecem praticamente parados. Normalmente é definida por dois parâmetros: tempo mínimo necessário para ser considerada uma fixação (100 ms) e amplitude do movimento máximo aceito para ser considerada uma fixação (raio de 50 pixels, quadrado de 40x40 pixels ou dois graus), mas também pode ser definida em relação à sua velocidade máxima (menor que 100 graus/segundo), dependendo do algoritmo usado para identificar as fixações (GIANNOTTO, 2010).

- Transição: Também conhecida como sacada, representa o caminho percorrido entre uma fixação e outra. Grosso modo, é cada vez que o olhar deixa uma área de interesse e se locomove para outra. A sacada pode se locomover da esquerda para a direita ou da direita para esquerda, sendo que nesse caso recebe o nome de "regressão".

- Visitas: Cada vez que o olhar do participante adentra uma área de interesse.

- Área de interesse $\left(\mathrm{AOI}^{6}\right)$ : Áreas delimitadas pelo pesquisador sobre a qual incidirá a análise. Em tradução geralmente se delimitam duas grandes áreas, uma dedicada ao texto-fonte e outra ao texto-alvo. Contudo, outras áreas dentro das duas citadas podem ser delimitadas, seja uma frase, uma oração, uma palavra ou mesmo um morfema. Dessas áreas são extraídos os dados estatísticos.

Com essas variáveis, é possível registrar a movimentação do olhar durante a tarefa e refazer o caminho percorrido pelos olhos dos participantes, desvendando possíveis padrões ou a ausência deles. Com a extração desses dados foi possível elaborar um modelo processual de (re)tradução, apresentado na seção 4 dedicada aos resultados.

\footnotetext{
${ }^{6}$ Do inglês áreas of interesting.
} 
Para o registro do fluxo de acessos entre as quatro AOI, este estudo se pautou em Hvelplund (2015). Em seu estudo, o autor analisa as transições e as fixações realizadas durante o processo de tradução para dublagem. Seu desenho experimental contempla quatro AOI: textofonte, texto-alvo, filme e dicionário. De sua pesquisa, utilizamos a metodologia para a contagem das transições.

Embora os objetivos e os tipos de tradução envolvidos nos dois estudos sejam diferentes, ambos gozam de similaridade quanto ao uso de quatro AOI, configuração incomum nos estudos processuais da tradução, tendo em vista que a maioria dos estudos contemplam duas grandes áreas, texto-fonte e texto-alvo. Compartilham, também, dados referentes ao rastreamento ocular. Hvelplund (2015) mostra, em seu estudo, que o processamento do textoalvo exige maior esforço cognitivo que quaisquer outros subprocessos de tradução para fins de dublagem, além de ser o mais demorado. Das quatro áreas que conformam o processo, o textoalvo foi a área na qual os participantes despenderam maior tempo e sobre a qual incidiu maior número e duração de fixações e visitas, dado corroborado neste estudo.

Na seção seguinte apresentamos a metodologia de coleta e análise dos dados.

\section{Metodologia}

Para o estudo foram recrutados catorze participantes entre professores de espanhol como língua estrangeira e estudantes de tradução no par espanhol-português, com mais de um ano e menos de quatro anos de experiência em tradução. Todos possuem nível intermediário ou superior de língua espanhola, comprovada com certificação ou, no caso dos estudantes, com estudos superiores a cinco semestres ou 300 horas $^{7}$ de língua espanhola.

O aparelho utilizado para rastreamento ocular foi um Tobii T60 com tela de LCD de 17 polegadas, 16 bits e resolução de 1280x1024 pixels. Para identificar e armazenar dados acerca do posicionamento dos olhos na tela do computador, foi utilizado o software Tobii Studio (versão 3.2.2). Com essa tecnologia, é possível mapear/gravar a movimentação do olhar durante a execução da tarefa de tradução, em tempo real, além de observar a produção textual. As variáveis de rastreamento ocular que fazem parte deste estudo são: Visitas (número e duração), fixações (número e duração) e fluxo de transições.

\footnotetext{
${ }^{7}$ O nível C1 (Domínio) compreende quatro módulos de 30 horas, cumprindo um total de 120h. É o nível anterior ao C2, último dos níveis na escala de proficiência- (cf. www.saopaulo.cervantes.es).
} 
O texto-fonte escolhido, um excerto de uma obra literária originalmente escrita em espanhol, variedade argentina, possui 98 palavras e data de 1946. As traduções desse excerto têm suas publicações datadas de 2005 e 2013 e possuem, respectivamente, 99 e 92 palavras. Utilizou-se fonte tamanho 15 e espaçamento entrelinhas de 1,5 nos três textos do experimento. O texto-fonte foi disponibilizado em uma janela na parte superior da tela do computador. As traduções foram disponibilizadas em duas janelas - à esquerda e à direita -, às quais a tradução mais antiga (T1) e a tradução mais recente (T2) foram distribuídas aleatoriamente para cada participante. Ao centro, entre as duas traduções, foi disponibilizado o espaço destinado à produção textual dos participantes, ou seja, o texto-alvo, conforme Figura 1 a seguir.

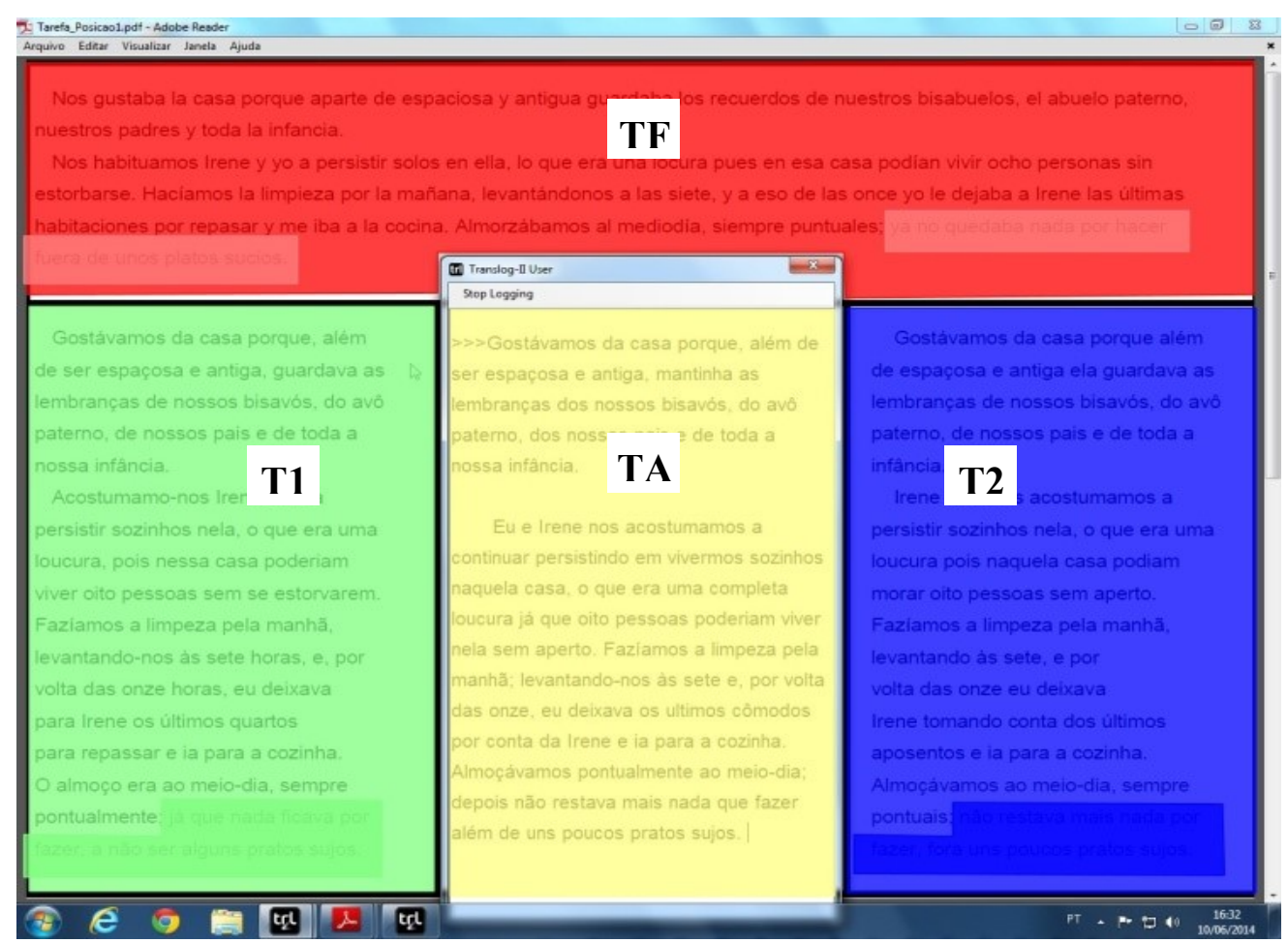

Figura 1. Print screen da tela do Tobii Studio 3.2.2 com as áreas de interesse (AOI). Nota: $\mathrm{TF}=$ texto-fonte, $\mathrm{TA}=$ texto-alvo; $\mathrm{T} 1=$ tradução $1 ; \mathrm{T} 2=$ tradução 2.

Fonte: Malta (2015, p. 62).

As áreas coloridas representadas na Figura 1 são denominadas área de interesse (AOI), a partir das quais foram extraídos os dados de rastreamento ocular. A tarefa foi realizada em duas configurações, com a T1 ora no lado esquerdo ora no lado direito. Os participantes catalogados com números ímpares realizaram a tarefa na configuração 1 (T1 do lado esquerdo), enquanto os participantes catalogados com números pares na configuração 2 (T1 lado direito). 
Para participar do experimento, todos os participantes preencheram um questionário demográfico disponibilizado on line e assinaram um termo de consentimento livre e esclarecido. Para amenizar o estranhamento dos participantes com o ambiente do texto, realizou-se teste de cópia que consistia na cópia simples de um texto. Ressaltamos que o leiaute do teste de cópia é idêntico ao da coleta definitiva, à exceção dos textos.

Conforme relatado anteriormente, as variáveis analisadas neste artigo são: número e duração de fixações ( $(\geq 180$ milissegundos), número e duração de visitas e número de transições. Posteriormente, procedeu-se com a extração dos dados relativos às transições e visitas e às fixações, adotando-se como filtro o padrão do Tobii Studio I-VT (velocity-threshold identification). O referido filtro classifica o movimento do olhar em três categorias: fixações, sacadas e indeterminado (unclassified). Foram descartados todos os participantes que apresentaram taxa de rastreamento ocular inferior a $70 \%$, ou seja, aqueles que ficaram menos de $70 \%$ do tempo olhando para a tela do computador (gaze time on screen).

Os dados foram exportados para tabelas do Excel e organizados para a leitura por outros programas estatísticos como o R, versão 3.1.1 e o SPSS (Statistical Package for the Social Sciences) ${ }^{8}$ versão 17 . Com o programa $\mathrm{R}$, foram realizados os testes de normalidade de Shapiro Wilk e os testes comparativos (i.e., teste T e Wilcoxon pareados); com o SPSS, foram realizados os testes de correlação. Na seguinte seção apresentamos os resultados da pesquisa.

\section{Resultados}

Esta seção está estruturada em três subseções. Na primeira são apresentados os dados referentes às fixações, depois às visitas e, por último, os referentes às transições. Nos dois primeiros casos são elencados tanto o número quanto a duração de cada variável em cada AOI. Já no terceiro caso, apresenta-se o caminho percorrido pelo olhar dos participantes em uma tarefa inédita envolvendo a (re)tradução.

\subsection{As fixações}

\footnotetext{
8 O software passou a se chamar IBM SPSS. Disponível em: http://www-01.ibm.com/software/analytics/spss/ e http://semiologiamedica.blogspot.com.br/2009/10/o-spss-statistical-package-for-social.html. Acesso em: 10 nov. 2015. Neste estudo, utiliza-se somente a sigla SPSS.
} 
Registrou-se um total de 15.559 fixações em todas as AOI. O maior número de fixações foi registrado na área do texto-alvo, com 6.438 (41,38\%); seguida do texto-fonte, com 5.934 (38,14\%); da tradução 2 (mais recente), com 1638 (10,85\%); e da tradução 1 (mais antiga), com 1.499 fixações (9,63\%). Observe-se que os números, absoluto e percentual, registrados nas traduções são bastante inferiores aos registrados nas áreas do texto-fonte e do texto-alvo, o que indica maior privilégio da atenção visual nessas áreas, sendo que o texto-fonte é onde se registra maior atenção visual com fixações.

Contudo, quando os participantes são analisados separadamente, tem-se que oito tiveram maior número de fixações no texto-fonte (P05, P06, P07, P08, P10, P14, P12 e P13) e seis no texto-alvo (P01, P02, P03, P04, P09, e P11). Mesmo assim, ao aplicar os testes estatísticos, observam-se diferenças significativas entre as áreas, conforme Tabela 1 a seguir:

Tabela 1. Resultados estatísticos referentes ao número de fixações.

\begin{tabular}{llll}
\hline Combinações & & \multicolumn{1}{c}{ Resultados } & \\
\hline TF-T1 & $t(13)=12,802$ & $p=0,000009583$ & TF $>$ T1 \\
TF-T2 & $t(13)=13,6018$ & $p=0,000004587$ & TF $>$ T2 \\
TA-T1 & $\mathrm{V}=0$ & $p=0,0001221$ (TA np) & TA $>$ T1 \\
TA-T2 & $\mathrm{V}=0$, & $p=0,0001221$ (TA np) & TA $>$ T2 \\
TF-TA & $\mathrm{V}=54$ & $n s$ & TF $<$ TA \\
T1-T2 & $t(13)=1,0018$ & $n s$ & T1<T2 \\
\hline
\end{tabular}

Nota: $\mathrm{np}=$ não paramétrico; $\mathrm{ns}=$ não significativo; $\mathrm{TA}=$ texto-alvo; $\mathrm{TF}=$ texto-fonte; $\mathrm{T} 1=$ tradução $1 ; \mathrm{T} 2=$ tradução 2 Fonte: Malta (2015, p. 120).

O número de fixações registrado no TF é muito superior ao número registrado nas duas traduções prévias, o mesmo ocorrendo na comparação entre o TA e as traduções T1 e T2. Em contrapartida, não há diferenças significativas entre o número de fixações registrado no TF em comparação com o TA e na T1 em comparação com a T2. O resultado parece indicar que as fixações de TF e TA, de um lado, e as de T1 e T2, de outro, podem ser da mesma natureza, o mesmo não ocorrendo na comparação ST[T1-T2] e TT[T1-T2]. Esses dados representam que houve maior concentração de atenção visual nas áreas do TF e do TA, o que significa que a relação de tradução tida como comum entre as duas áreas prevalece sobre qualquer outra relação comas as traduções que serviram de insumo.

Com relação à duração das fixações tem-se praticamente o mesmo quadro. Em média os participantes despenderam 208 milissegundos (ms) em cada fixação. O TA novamente possui maior média, $251 \mathrm{~ms}$, seguido pelo TF com duração média de $216 \mathrm{~ms}$. Com relação às traduções prévias, a T2 possui média superior à da T1 - 192 ms e 173 ms, respectivamente -, 
porém menor que aquela registradas no TT e no ST. Estes dados corroboram os primeiros referentes ao número de fixações, que indicam maior atenção visual no TA, no TF, na T2 e na T1, sucessivamente. Ao aplicar os testes estatísticos, a única combinação na qual o resultado não apresenta significância é a T1-T2, conforme mostra a Tabela 2.

Tabela 2. Resultados dos estatísticos referentes à variável duração das fixações.

\begin{tabular}{lll}
\hline Combinação & \multicolumn{2}{c}{ Resultados } \\
\hline ST-TT & $t(13)=2,7656$ & $p=0,01605$ \\
T1-T2 & $t(13)=1,0018$ & $n s$ \\
ST-T1 & $t(13)=5,0551$ & $p=0,0002205$ \\
ST-T2 & $t(13)=3,7724$ & $p=0,002326$ \\
TT-T1 & $t(13)=7,2171$ & $p=0,006776$ \\
TT-T2 & $t(13)=5,3568$ & $p=0,0001305$ \\
\hline
\end{tabular}

Nota: $n s=$ não significativo; $\mathrm{TA}=$ texto-alvo; $\mathrm{TF}=$ texto-fonte; $\mathrm{T} 1=$ tradução $1 ; \mathrm{T} 2=$ tradução 2 . Fonte: Malta (2015, p. 127).

O resultado exposto na Tabela 2 revela a diferença entre as fixações registradas no TA e no TF, e entre as fixações registradas nessas áreas e nas traduções 1 e 2 , o que pode envolver a intenção do participante ao acessar uma área e fixar, por mais ou por menos tempo, o olhar em determinada parte ou palavra, como ocorreu com as combinações TF-TA - onde TA tem média de duração da fixação maior que TF-, TF-T1, com $p<0,05(\mathrm{TF}=216>\mathrm{T} 1=172)$, TF$\mathrm{T} 2,(p<0,05, \mathrm{TF}=216>\mathrm{T} 2=192), \mathrm{TA}-\mathrm{T} 1(p<0,05, \mathrm{TA}=251>\mathrm{T} 1=172)$ e TA-T2, $(p<$ $0,05, \mathrm{TF}=251>\mathrm{T} 2=192)$.

\subsection{As visitas}

O comportamento do olhar concernente às visitas é similar ao registrado quando das fixações. Com um total de 2.789 visitas, registrou-se maior acesso ao TA, com 1.152 visitas (41,31\%), seguido, nesta ordem, do TF, com 982 (35,21\%); da T1, com 337 (12,08\%); e da T2, com 318 visitas $(11,4 \%)$.

Ao aplicar o teste $\mathrm{T}$ pareado, a diferença entre o número de visitas em cada AOI é significativa em diferentes casos: entre TF e T1, TF e T2, TA e TF, TA e T1, e TA e T2, todas $\operatorname{com} p<0,05$. No entanto, as diferenças não apresentaram nível de significância quando a relação envolve somente as traduções, ou seja, T1 e T2.

Tabela 3. Resultados estatísticos relativos à variável número de visitas. 


\begin{tabular}{lccc}
\hline Combinação & & Resultados & \\
TF-T1 & $t(13)=6.6571$ & $p=0,01571$ & $\mathrm{TF}>\mathrm{T} 1$ \\
TF-T2 & $t(13)=6.7485$ & $p=0,01365$ & $\mathrm{TF}>\mathrm{T} 2$ \\
TA-TF & $t(13)=-3.8572$ & $p=0,00198$ & $\mathrm{TA}>\mathrm{TF}$ \\
TA-T1 & $t(13)=-8.7056$ & $p=0,000000876$ & $\mathrm{TA}>\mathrm{T} 1$ \\
TA-T2 & $t(13)=-8.9945$ & $p=0,0006063$ & $\mathrm{TA}>\mathrm{T} 2$ \\
T1-T2 & $t(13)=0,5669$ & $n s$ & $\mathrm{~T} 1>\mathrm{T} 2$ \\
\hline
\end{tabular}

Nota: ns=não significativo; TA=texto-alvo; TF=texto-fonte; T1=tradução 1; T2=tradução 2 .

Fonte: Malta (2015, p. 89).

No que tange à duração das visitas, foi despendido, em média, o tempo de $1641 \mathrm{~ms}$ em cada visita. A AOI em que a média de duração da visita é maior é o TA (2438 ms), seguido pelo TF $(1797 \mathrm{~ms})$, pela T2 $(1317 \mathrm{~ms})$ e pela T1 $(1013 \mathrm{~ms})$. No caso dessa variável, todos os testes estatísticos foram significativos, conforme mostrado na Tabela 4.

Tabela 4. Resultados estatísticos referentes à variável duração das visitas.

\begin{tabular}{cccc}
\hline Combinações & \multicolumn{3}{c}{ Resultados dos testes de comparação } \\
\hline TFA1 & $\mathrm{V}=105$ & $p=0,001094(\mathrm{TF}=\mathrm{np})$ & $\mathrm{TF}>\mathrm{T} 1$ \\
TFA2 & $\mathrm{V}=89,5$ & $p=0,02191(\mathrm{TF}=\mathrm{np})$ & $\mathrm{TF}>\mathrm{T} 2$ \\
$\mathrm{~T} 1 \mathrm{~T} 2$ & $t(13)=-2,9399$ & $p=0,01149$ & $\mathrm{~T} 1<\mathrm{T} 2$ \\
TATF & $\mathrm{V}=2$ & $p=0,0003662$ & $\mathrm{TA}>\mathrm{TF}$ \\
TAT1 & $t(13)=-8,4741$ & $p=0,001184$ & $\mathrm{TA}>\mathrm{T} 1$ \\
TAT2 & $t(13)=-5,4601$ & $p=0,0001093$ & $\mathrm{TA}>\mathrm{T} 2$ \\
\hline
\end{tabular}

Nota: $\mathrm{np}=$ não paramétrico; $\mathrm{ns}=$ não significativo; $\mathrm{TA}=$ texto-alvo; $\mathrm{TF}=$ texto-fonte; $\mathrm{T} 1=$ tradução $1 ; \mathrm{T} 2=$ tradução 2 Fonte: Malta (2015, p. 98)

Os dados da Tabela 4 mostram que há diferenças significativas em todas as combinações. Aventa-se a hipótese de as visitas possuírem características diferenciadas em consequência de seus propósitos, também diferentes. Outra possível explicação para as diferenças é o tipo de tarefa proposta neste estudo, uma tarefa inédita, em cujo formato os participantes não possuem qualquer experiência, o que os torna participantes novatos, podendo resultar em um comportamento errático.

Sinteticamente, tanto os dados relativos às fixações como os relativos às visitas aponta para maior atenção visual no TA e no TF em detrimento das traduções. Esse comportamento gera a ideia de que as traduções foram utilizadas como apoio para a nova tradução, prevalecendo a relação entre o TF e o TA. Na seguinte subseção apresentam-se os dados referentes às transições.

\subsection{As transições}


Antes de apresentar os dados concernentes às transições, é importante demonstrar como eles foram extraídos. Um dos propósitos do estudo em voga era justamente caracterizar o processo de (re)tradução. Para tanto, foi necessário acessar cada registro de mudança do olhar de entre as AOI, delimitando, assim, o caminho percorrido pelo olhar dos participantes. Como relatado anteriormente, tivemos como base o estudo de Hvelplund (2015) sobre o processo de tradução para dublagem.

Os dados crus foram exportados com o Tobii Studio 3.2.2 e organizados em tabelas do Excel. Nas tabelas, os dados foram dispostos de forma que somente os números referentes às transições, marcadas pela existência (1) ou não (2) de fixações em cada AOI, fossem considerados. Nesse processo, foram excluídas as células vazias e aquelas para as quais o valor fosse "0" nas quatro AOI. Também foram excluídos os dados referentes às sacadas e os indeterminados (unclassified), possibilitando a criação de uma sequência linear de acessos, conforme exemplo adaptado do participante P01.

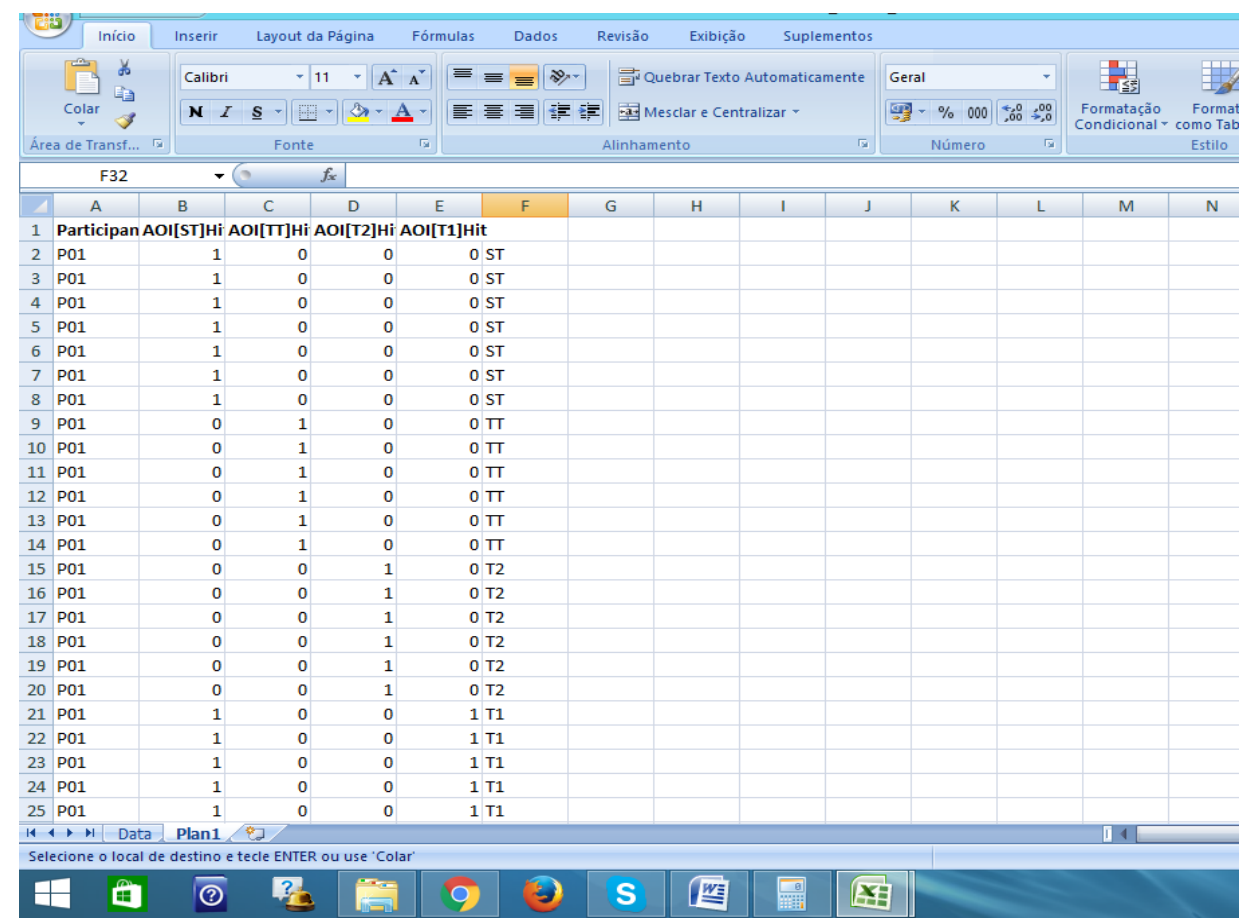

Figura 2 - Tela do Excel com exemplo de contagem de fixações e criação de sequência linear de acessos de P01 (adaptado).

Nota: ST=texto-fonte; TT=texto-alvo; T1=tradução 1; T2=tradução 2.

Fonte: Malta (2015, p. 106) 
Posteriormente, realizou-se uma contagem de cada transição, a partir da qual uma sequência de transições foi gerada. Por fim, a contagem das transições foi organizada em uma planilha do Excel, conforme Figura 3.

Figura 3. Print screen da tela do Excel com o registro das transições por participante e por AOI.

\begin{tabular}{|c|c|c|c|c|c|c|c|c|c|c|c|c|c|c|c|c|c|c|}
\hline & B2 & $\cdot$ & 6 & $f_{x}$ & & & & & & & & & & & & & & \\
\hline 4 & A & B & C & D & E & $\mathrm{F}$ & G & H & 1 & J & K & L & M & $\mathrm{N}$ & 0 & P & Q & $\mathrm{R}$ \\
\hline 1 & & TF-TF & TF-T1 & TF-T2 & TF-TA & T1-TF & T1-T1 & T1-T2 & T1-TA & T2-TF & $\mathrm{T} 2-\mathrm{T} 1$ & T2-T2 & T2-TA & TA-TF & TA-T1 & TA-T2 & TA-TA & \\
\hline 2 & P3 & 336 & 0 & 0 & 81 & 2 & 16 & 1 & 5 & 0 & 0 & 20 & 5 & 78 & 8 & 4 & 825 & \\
\hline 3 & P5 & 260 & 2 & 3 & 35 & 2 & 48 & 0 & 13 & 3 & 2 & 36 & 4 & 35 & 11 & 6 & 299 & \\
\hline 4 & P16 & 7464 & 6 & 8 & 59 & 5 & 1325 & 4 & 21 & 7 & 7 & 1998 & 25 & 61 & 17 & 27 & 9645 & \\
\hline 5 & P17 & 4670 & 5 & 12 & 30 & 3 & 412 & 3 & 7 & 6 & 2 & 2059 & 21 & 37 & 6 & 14 & 5790 & \\
\hline 6 & P20 & 3693 & 4 & 7 & 25 & 3 & 1027 & 6 & 10 & 5 & 3 & 780 & 9 & 29 & 12 & 4 & 5480 & \\
\hline 7 & P21 & 6898 & 10 & & 34 & 16 & 3426 & 5 & 17 & 7 & 9 & 2899 & 7 & 29 & 19 & 10 & 5252 & \\
\hline 8 & P23 & 4875 & 9 & 7 & 23 & 9 & 2245 & 7 & 16 & 9 & 9 & 2275 & 14 & 21 & 14 & 18 & 4475 & \\
\hline 9 & P24 & 5952 & 8 & 4 & 82 & 11 & 792 & 0 & 13 & 1 & 1 & 902 & 9 & 81 & 16 & 7 & 5581 & \\
\hline 10 & P28 & 4401 & 13 & & 52 & 14 & 1591 & 12 & 12 & 10 & 9 & 1761 & 24 & 50 & 15 & 22 & 8065 & \\
\hline 11 & P30 & 5713 & 3 & 4 & 97 & 3 & 93 & 2 & 3 & 2 & 2 & 479 & 5 & 99 & 3 & 4 & 5352 & \\
\hline 12 & P10 & 6099 & 5 & 0 & 61 & 6 & 1343 & 3 & 20 & 8 & 0 & 1465 & 10 & 52 & 24 & 15 & 10094 & \\
\hline 13 & P13 & 6080 & 7 & 6 & 52 & 9 & 1028 & 9 & 9 & 9 & 3 & 1615 & 12 & 47 & 17 & 9 & 7727 & \\
\hline 14 & P22 & 6643 & 5 & 3 & 53 & 7 & 1489 & 7 & 10 & 3 & 6 & 1821 & 16 & 51 & 12 & 15 & 7645 & \\
\hline 15 & P27 & 3409 & 5 & 5 & 42 & 9 & 292 & 10 & 4 & 4 & 10 & 580 & 11 & 38 & 8 & 10 & 2593 & \\
\hline 16 & TOTAL & 66493 & 82 & 76 & 726 & 99 & 15127 & 69 & 160 & 74 & 63 & 18690 & 172 & 708 & 182 & 165 & 78823 & \\
\hline 17 & Média & $4749,5^{\prime \prime}$ & 5,857143 & 5,428571 & 1' 51,85714 & 7,071429 & 1080,5 & 4,928571 & 11,42857 & 5,285714 & 4,5 & 1335 & 12,28571 & 50,57143 & 13 & 11,78571 & 5630,214 & \\
\hline 18 & DP & $2222,73^{\prime}$ & 3,370851 & ' 3,390355 & $5^{\prime} 22,64053$ & 4,480115 & 950,524 & 3,75119 & 5,680118 & 3,196839 & ' 3,674235 & '877,9922" & 6,921856 & '22,42497' & '5,602197" & 7,083877 & 2945,27 & \\
\hline 19 & & & & & & & & & & & & & & & & & & \\
\hline 20 & & 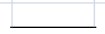 & & & & & & & & & & & & & & & & \\
\hline
\end{tabular}

A relação de tradução impressa entre TF e TA fica mais evidente quando observada a quantidade de transições entre essas AOI e sua comparação com o número de transições realizadas entre elas e a T1 e a T2 (Figura 3). As transições realizadas do TF ao TA e na direção inversa correspondem a $29,54 \%$ e $28,87 \%$ dos dados. Além disso, o número de transições do TA para as traduções é mais que o dobro do número de transições que partem do TF para essas mesmas traduções (347 e 158, respectivamente). Com relação à área das traduções, observa-se que o acesso à T2 é menor que o acesso à T1, independentemente da origem da transição. Esse comportamento pode ser um indicador de que as traduções, por serem bem menos acessadas, cumprem a função de fonte de consulta na direção TA[T1-T2] ou averiguação do que foi processado na direção TA[T1-T2].

Na direção T1[TF-TA], foram registradas 259 transições, sendo 99 para o TF e 160 para o TA. Na direção T2[TF-TA], foram registradas 246 transições, dessas 63 para o TF e 172 para o TA. Como comentado anteriormente, o TA é o destino mais frequente quando a transição tem como origem uma das traduções, o que significa que a relação de tradução entre TF-TA, pelo número de transições, é maior que a entre as outras AOI.

Esses dados corroboram o estudo de Hvelplund (2015), o qual apontou número elevado de transições entre TF e TA. Contudo, como este trabalho e o de Hvelplund (2015) possuem objetos de estudo diferentes, o nível de comparabilidade entre os comportamentos é 
limitado, embora o acesso às traduções, no caso da presente pesquisa, também tenha sido mais esporádico se comparado com os acessos gerados entre TF e TA. Com os dados devidamente extraídos e organizados, elaborou-se a matriz de fluxos de acessos em um processo de (re)tradução, representado na Tabela 5 a seguir.

\begin{tabular}{|c|c|c|c|c|}
\hline & $\rightarrow \mathrm{TF}$ & $\rightarrow \mathrm{TA}$ & $\rightarrow \mathrm{T} 1$ & $\rightarrow \mathrm{T} 2$ \\
\hline $\mathrm{TF} \rightarrow$ & - & $726(28,18 \%)$ & $82(3,18 \%)$ & $76(2,95 \%)$ \\
\hline $\mathrm{TA} \rightarrow$ & $708(27,48 \%)$ & - & $182(7,07 \%)$ & $165(6,41 \%)$ \\
\hline $\mathrm{T} 1 \rightarrow$ & $99(3,84 \%)$ & $160(6,21 \%)$ & - & $69(2,68 \%)$ \\
\hline $\mathrm{T} 2 \rightarrow$ & $74(2,87 \%)$ & $172(6,68 \%)$ & $63(2,45 \%)$ & - \\
\hline
\end{tabular}

Fonte: Malta (2015, p. 112).

O número de transições realizadas entre o Tf e o TA (1.434 ou 55,66\%), nas duas direções, é maior que a soma de todas as transições realizadas nas outras combinações nas quais há alguma relação com as traduções (1.142 ou 44,33\%). Dessa forma, a configuração que emana dos dados contempla uma forte relação entre TF e TA e menor número de transições essas áreas e as áreas das traduções (T1 e T2).

Contudo, vale ressaltar que as transições realizadas intra-áreas são distintas das transições interáreas. Enquanto as primeiras envolvem processamento do tipo compreensão e expressão (TF e TA), as interáreas, principalmente as que envolvem as traduções, são caracterizadas, principalmente, pela busca de palavras, seja para auxiliar na elaboração da nova tradução seja para a confirmação de uma informação já processada. O resultado dos testes estatísticos não apontou diferenças significativas quando a comparação é realizada com dados de transições efetuadas dentro das próprias áreas, ou seja, TFTF [TATA, T1T1, T2T2], TATA[T1T1, T2T2] e T1T1-T2T2. Entre colchetes estão as combinações com as quais as transições realizadas na combinação que está fora do colchete são comparadas estatisticamente, não havendo diferença entre, por exemplo, as transições realizadas dentro do TF em comparação com as realizadas dentro do TA, da T1 e da T2. Tampouco houve diferenças significativas quando da comparação entre TATA-TFTF e TFTF-TFTA, sendo esta última interáreas. No entanto, quando as comparações têm por objeto as transições interáreas, algumas combinações apresentam diferenças significativas. Pode-se inferir, com isso, que o tipo de transição realizado dentro da mesma área (e.g., TFTF, TATA) é diferente do realizado entre duas áreas distintas (TF-T1). 
O resultado da comparação entre as combinações T1T2-T2T1 e T2T1-T1T2 também não é significativo, embora o tipo de transição seja diferente do registrado no primeiro caso. Enquanto no primeiro caso há uma comparação entre transições diferentes (inter e intra-áreas), no segundo as transições são somente do tipo interáreas (T1T2-T2T1). O resultado, nesse caso, deve-se ao fato de as transições terem sido realizadas entre as traduções (T1 e T2), áreas em que o número de visitas é limitado e específico, e pode indicar que as transições a essas áreas possuem características parecidas. Os resultados do teste $\mathrm{T}$ pareado e de Wilcoxon estão expostos na Tabela 6 a seguir.

Tabela 6. Resultados estatísticos referentes às transições interáreas.

\begin{tabular}{|c|c|c|c|c|c|}
\hline \multicolumn{3}{|c|}{$\begin{array}{l}\text { Combinações com origem da transição em TF e } \\
\text { TA }\end{array}$} & \multicolumn{3}{|c|}{$\begin{array}{l}\text { Combinações com origem da transição em T1 e } \\
\text { T2 }\end{array}$} \\
\hline TF-T1 & TF-T2 & $t(13)=0,5043 ; n s$ & $\mathrm{~T} 1-\mathrm{TF}$ & $\mathrm{T} 1-\mathrm{T} 2$ & $t(13)=1,9868 ; n s$ \\
\hline TF-T1 & TF-TA & $t(13)=-7,2435 ; n s$ & $\mathrm{~T} 1-\mathrm{TF}$ & T1-TA & $t(13)=2,7021 ; p=0,01812$ \\
\hline TF-T1 & TA-TF & $t(13)=-7,0657 ; n s$ & $\mathrm{~T} 1-\mathrm{TF}$ & $\mathrm{T} 2-\mathrm{TF}$ & $t(13)=1,6229 ; n s$ \\
\hline TF-T1 & TA-T1 & $t(13)=-5,4755 ; p=0,0001065$ & $\mathrm{~T} 1-\mathrm{TF}$ & $\mathrm{T} 2-\mathrm{T} 1$ & $\mathrm{~V}=58, P=0,02843$ \\
\hline TF-T1 & TA-T2 & $t(13)=-3,7499 ; p=0,002428$ & $\mathrm{~T} 1-\mathrm{TF}$ & T2-TA & $t(13)=-2,6282 ; p=0,02086$ \\
\hline TF-T2 & TF-TA & $t(13)=-7,0109 ; \mathrm{ns}$ & $\mathrm{T} 1-\mathrm{T} 2$ & $\mathrm{~T} 2-\mathrm{TF}$ & $t(13)=-0,445 ; n s$ \\
\hline TF-T2 & TA-T1 & $t(13)=-4,1513 ; p=0,001138$ & $\mathrm{~T} 1-\mathrm{T} 2$ & $\mathrm{~T} 2-\mathrm{T} 1$ & $\mathrm{~V}=45 ; n s$ \\
\hline TF-T2 & TA-T2 & $t(13)=3,69,33 ; p=0,002704$ & $\mathrm{~T} 1-\mathrm{T} 2$ & T2-TA & $t(13)=-3,4568 ; p=0,004252$ \\
\hline TF-TA & TA-TF & $t(13)=1,1725 ; n s$ & T1-TA & $\mathrm{T} 2-\mathrm{TF}$ & $t(13)=4,709 ; p=0,0004086$ \\
\hline TF-TA & TA-T1 & $t(13)=6,0235 ; n s$ & T1-TA & $\mathrm{T} 2-\mathrm{T} 1$ & $\mathrm{~V}=98,5, P=0,004257$ \\
\hline TF-TA & TA-T2 & $t(13)=5,963 ; n s$ & T1-TA & T2-TA & $t(13)=-0,4235 ; n s$ \\
\hline TA-TF & TA-T1 & $t(13)=-5,732 ; n s$ & $\mathrm{~T} 2-\mathrm{TF}$ & $\mathrm{T} 2-\mathrm{T} 1$ & $\mathrm{~V}=29 ; n s$ \\
\hline TA-TF & TA-T2 & $t(13)=5,8087 ; n s$ & $\mathrm{~T} 2-\mathrm{TF}$ & T2-TA & $t(13)=4,5541 ; p=0,0005411$ \\
\hline TA-T1 & TA-T2 & $t(13)=0,6504 ; n s$ & $\mathrm{~T} 2-\mathrm{T} 1$ & T2-TA & $\mathrm{V}=2,5 ; p=0,001873$ \\
\hline $\mathrm{TF}-\mathrm{TF}$ & TA-TA & $t(13)=-1,9944 ; n s$ & $\mathrm{~T} 1-\mathrm{T} 1$ & $\mathrm{~T} 2-\mathrm{T} 2$ & $t(13)=-1,8783 ; n s$ \\
\hline TF-TF & TF-TA & $t(13)=-7,9151 ; n s$ & - & - & - \\
\hline
\end{tabular}

Os dados específicos das transições realizadas entre as áreas representam o tipo de padrão de alternância mais frequente. Segundo Hvelplund (2015), as transições são as responsáveis pelo fluxo do processamento (processing flow), ou seja, as diferentes tarefas que o tradutor executa quando muda de área de interesse. Estudos mostram que o TA é a área sobre a qual recai a maior parcela da atenção visual (cf. HVEPLUND, 2011; 2015; JAKOBSEN; JENSEN, 2008, PAVLOVIĆ; JENSEN, 2009, MALTA, 2015). No caso da (re)tradução, $28,18 \%$ das transições tiveram o TF como origem e o TA como destino. No caso deste estudo, o fluxo típico de processamento é o representado na Tabela 7. 
Tabela 7. Matriz de transição entre ST, TT, T1 e T2 (em \%).

\begin{tabular}{|c|c|c|c|c|}
\hline & $\rightarrow \mathrm{TF}$ & $\rightarrow \mathrm{TA}$ & $\rightarrow \mathrm{T} 1$ & $\rightarrow \mathrm{T} 2$ \\
\hline $\mathrm{TF} \rightarrow$ & - & 82,13 & 9,28 & 8,59 \\
\hline $\mathrm{TA} \rightarrow$ & 67,11 & - & 17,25 & 15,64 \\
\hline $\mathrm{T} 1 \rightarrow$ & 30,18 & 48,78 & $\begin{array}{ll}- & \text { - }\end{array}$ & 21,04 \\
\hline $\mathrm{T} 2 \rightarrow$ & 23,94 & 55,67 & 20,39 & - \\
\hline
\end{tabular}

De acordo com a Tabela 7, o processo tem início com a leitura do TF, depois a atenção se dirige para o TA em 82,13\% dos casos. Em seguida, a atenção ou regressa para o TF, possibilidade de $67,11 \%$, ou se bifurca, com maior chance de mover-se para a T1 $(9,28 \%)$, ou para a T2 (8,59\%). Independente se o destino é uma ou outra tradução, a maior possibilidade é que a atenção retorne para o TT, 55,67\% dos casos se a origem é a T2, e 48,78\% se a origem da transição é a T1. A atenção visual retorna ao ST em 30,18\% dos casos em que ela parte da T1, e em 23,94\% dos casos em que ela tem como origem a T2. Similarmente ao fluxo descrito no estudo de Hvelplund (2015), aferimos que o TA também funciona como elo de processamento de informação durante o processo de (re)tradução. Visualmente, o processo de (re)tradução pode ser visualizado na Figura 4, cujos mapas de calor (a) e gaze plot (b) mostram os locais com maior incidência da atenção visual dos participantes.

a)

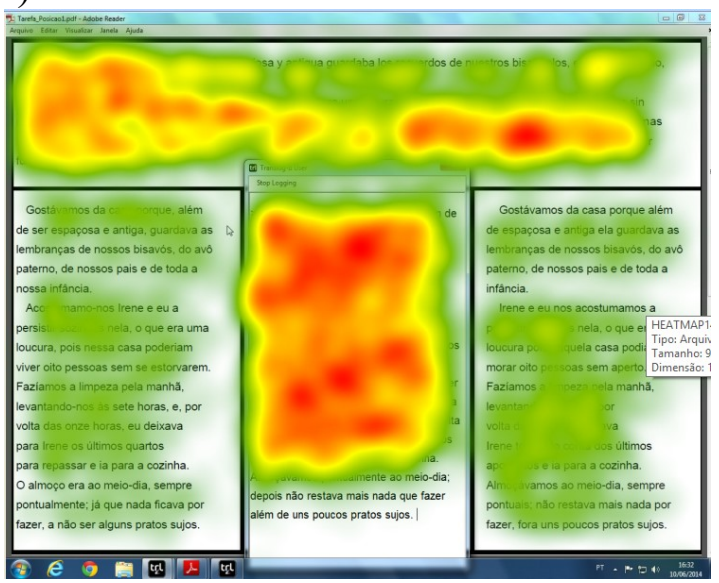

b)

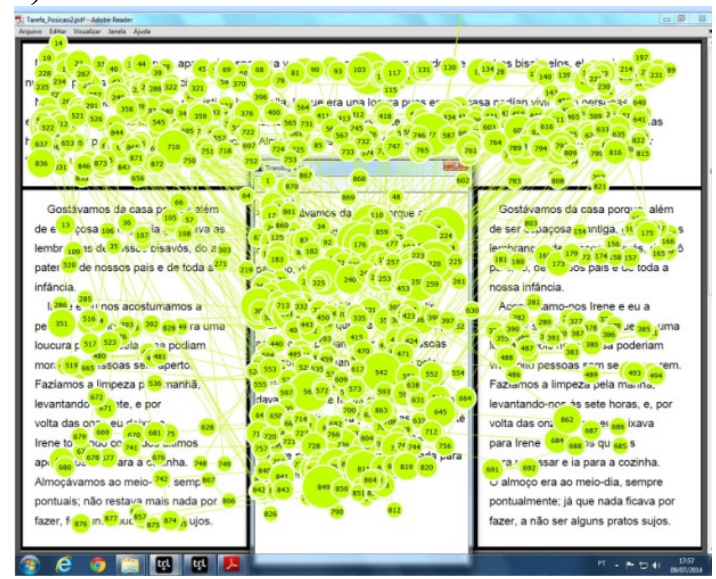

Figura 4. Mapa de calor e gaze plot do processo de (re)tradução.

As cores representadas no mapa de calor representado na Figura 4 "a" indicam as áreas nas quais houve maior incidência de atenção visual. As tonalidades de cores, que vão do verde ao vermelho, indicam uma escala crescente no número de visitas, ou seja, de verde a transparente menor concentração de visitas, sacadas e fixações, e de amarelo a vermelho, maior 
atenção visual. Já no lado "b" da Figura 4, observa-se o caminho percorrido pelo olhar com a quantificação de fixações e sacadas (linhas entre as fixações), o que também comprova maior atenção visual no TA e no TF, e menor atenção nas áreas das traduções.

Fundamentados nos dados de rastreamento, modelou-se o processo de (re)tradução, representado na Figura 5 a seguir.

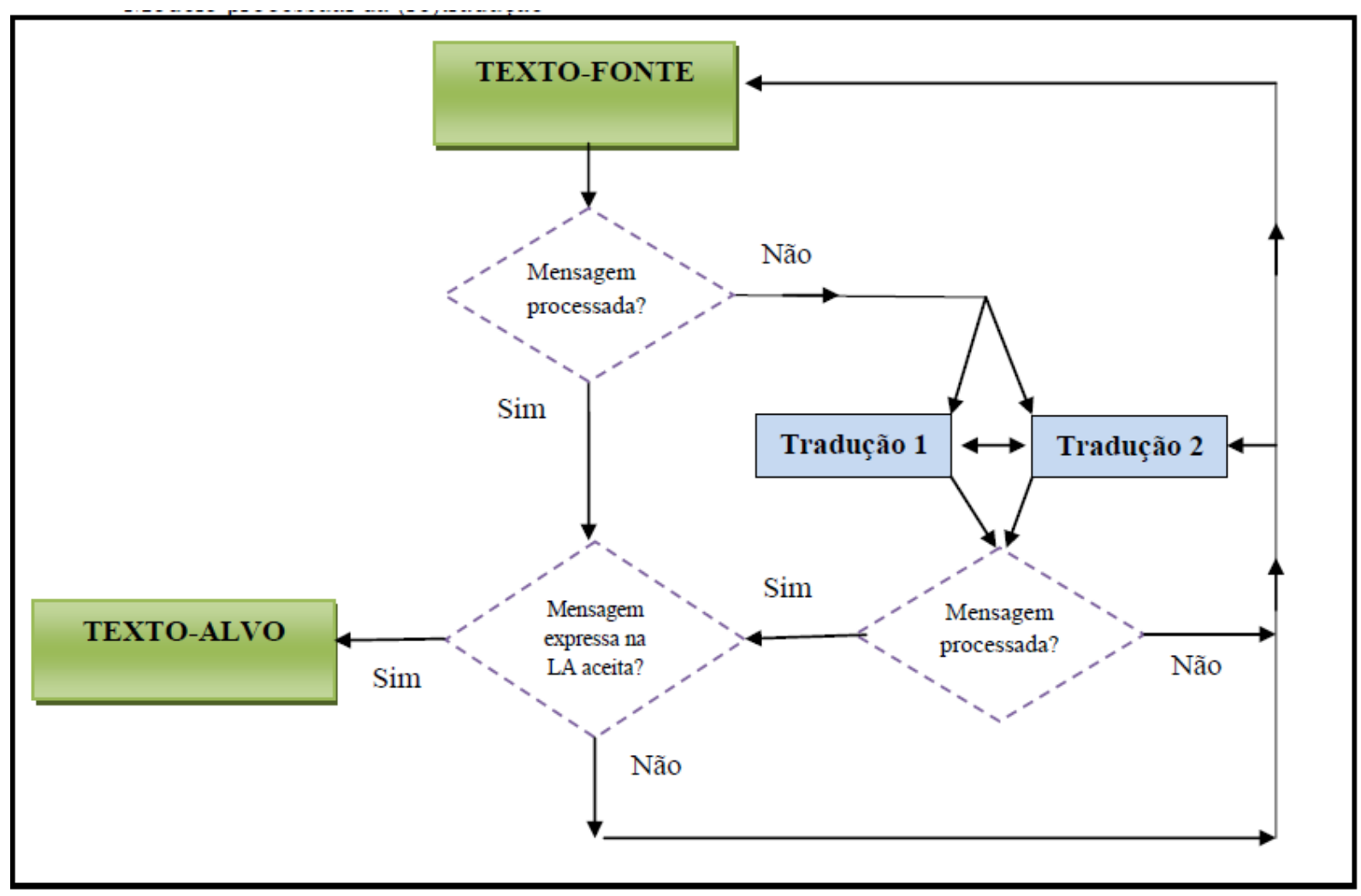

Figura 5. Modelo processual da (re)tradução. Fonte: Malta (2015, p. 189).

O modelo processual da (re)tradução, representado pela Figura 5, ilustra os possíveis caminhos percorridos em uma tarefa de (re)tradução, com base nos resultados obtidos dos dados de rastreamento ocular. Ao ler o TF, o participante tem basicamente dois caminhos: i) buscar auxílio nas traduções para a conversão da mensagem ainda não processada (total ou parcialmente) para a língua-alvo; ou ii) traduzir a mensagem e retornar às traduções para confirmar/contrastar a mensagem processada, o que pode ocorrer concomitantemente com a elaboração da nova tradução, ou nas fases de orientação e revisão. Paralelo aos dois caminhos, há o que vai de uma tradução prévia à outra, por meio do qual se poderá escolher entre as opções existentes nas traduções ou rechaçá-las, seja completamente, seja parcialmente, e elaborar a nova tradução. 
Dessa forma, chegou-se às duas definições de (re)tradução dentro de uma abordagem processual:

[como tarefa] Tarefa na qual o tradutor parte de um texto-fonte já traduzido para a mesma língua-alvo, podendo, ou não, utilizar a(s) tradução(ões) existente(s) desse mesmo texto-fonte, na produção da nova tradução. Nesse caso, as traduções existentes funcionam como apoio/insumo para a reformulação da nova tradução.

[como processo] Processo que envolve a produção de um texto-alvo, tendo por base o texto-fonte, além de uma ou mais traduções desse texto-fonte na mesma língua, ou não, que podem servir de suporte para a produção da nova tradução. (MALTA, 2015, p. 190)

Uma vez modelado o processo e definida a (re)tradução processualmente, passamos a tecer nossas considerações finais na próxima seção.

\section{Considerações finais}

Este artigo abordou o tema da retradução sob o viés processual dos Estudos da tradução. Os Estudos da Tradução como abordagem processual vêm nos últimos 15 anos incrementando e implementando metodologias de pesquisa de natureza empírico-experimental e contribuindo com dados para as diferentes atividades que seu nicho abrange.

Muitos processos foram descritos e analisados, desde os que envolvem a relação entre ST e TT da forma mais clássica, no caso das tarefas de tradução, até estudos de pós-edição monolíngue e bilíngue (i.e., sem e com acesso ao ST respectivamente). Agregam-se a esse contexto pesquisas sobre leitura para tradução e sobre as fases de orientação e revisão, sendo estas últimas mais carentes de pesquisas processuais de base empírica.

A (re)tradução é um tipo de tradução que possui características peculiares. Por envolver a tradução de um texto já traduzido, ultrapassa fronteiras e adentra terrenos próprios de outras atividades, como a revisão e a reedição, além de suscitar dúvidas, inclusive, referentes à autoria. Assim é a (re)tradução: polêmica; porém, mais que polêmica, necessária. Talvez essa capacidade ou propriedade de causar estranhamentos e suscitar os mais diferentes e antagônicos sentimentos com relação ao seu propósito seja uma característica herdada de sua principal matéria-prima, a literatura. Seja assimiladora ou domesticadora segundo Venutti (2002), seja estrangeirizadora (VENUTTI, 2002), a (re)tradução é a responsável por manter vivos os clássicos.

Distante da discussão acadêmica e filosófica sobre a legitimidade da HR, é inegável que o ato de (re)traduzir é tão ou mais corrente que o próprio ato de traduzir, como mostra o 
estudo de Paloposki e Koskinen (2010), no qual casos de retradução, reimpressão e reedição, são tão frequentes quanto os casos de novas tradução. No Brasil, não são raros os exemplos de obras traduzidas ao português que já foram reeditadas, relançadas, reimpressas e/ou retraduzidas. Do mundo hispânico, a obra La casa de los espíritus, de Isabel Allende, publicada no Chile em 1982, teve, em 2013, sua 43ª edição traduzida no Brasil (cf. MALTA, 2015; MALTA; RAEL, 2015). Tal como a obra de Allende, o Prêmio Nobel de Literatura (1982) Cien años de soledad, de Gabriel García Márquez (1967), foi traduzida para mais de 35 idiomas. No Brasil, com mais de 38 edições, a obra foi retraduzida por tradutores diferentes dentro da própria editora (Record), Eric Nepomuceno e Eliane Zaguri. Em 2009, o Brasil ganhou uma edição comemorativa dos 40 anos da obra. Segundo seu tradutor, o escritor Eric Nepomuceno, em entrevista à agência EFE reproduzida pelo Portal G1 ${ }^{9}$, trata-se de uma tradução completamente nova, já que a obra original tinha sido revisada pelo próprio García Márquez. Como água para chocolate, clássicos como Don Quijote, de Cervantes, com mais de 38 edições, Casa tomada, de Julio Cortázar, entre tantos outros títulos, para citar somente os do mundo hispânico, já foram alvo desse fenômeno chamado (re)tradução. Os estudos processuais não poderiam fazer vistas grossas a esse fenômeno tão comum no mundo da tradução.

Por tratar-se de um estudo exploratório de cunho experimental, algumas características podem influenciar negativamente nos resultados. O perfil dos participantes é um deles, já que não foram recrutados tradutores profissionais. Por outro lado, também é uma incógnita o processo que cada tradutor se auto delimita para realizar uma (re)tradução. Dessa forma, é possível que mesmo tradutores profissionais tenham um comportamento errático diante de uma tarefa ainda não explorada nos estudos com abordagem processual. Outro ponto que merece atenção é o que diz respeito ao par de línguas utilizado no experimento, espanhol>português. Sugere-se que novas pesquisas sejam realizadas com outros pares de línguas, os quais a proximidade tipológica e léxica seja menor que a do binômio utilizado neste estudo.

Este estudo enfocou, assim, o processo de (re)tradução como uma atividade cuja caracterização poderá oferecer subsídios para novas pesquisas, contribuindo para o estado da arte da abordagem processual, que passa a ter uma caracterização de um processo que ainda não havia sido descrito. Ademais, os resultados poderão ser úteis em outras ramas do campo

\footnotetext{
9 EFE. Brasil ganha edição comemorativa de "Cem anos de solidão". In: Portal G1, 13 out. 2009. Disponível em: http:/g1.globo.com/Noticias/PopArte/0,,MUL1339456-7084,00-BRASIL+GANHA+EDICAO+COME MORATIVA+DE+CEM+ANOS+DE+SOLIDAO.html. Acesso em: 22 nov. 2015.
} 
disciplinar, como a rama aplicada, em subáreas como a didática da tradução, possibilitando a formulação de unidades didáticas contribuindo com a formação de tradutores.

\section{Referências Bibliográficas}

BAKER, M.; MALMKJAER, K. Routledge encyclopaedia of translation studies. 1. ed. Londres: Routledge, 1998. http://dx.doi.org/10.4324/9780203359792

BENSIMON, P. Présentation. Palimpsestes, v. 13, p. IX-XIII, 1990

BERMAN, A. La retraduction comme espace de traduction. Palimpsestes, v. 13, p. 1-7, 1990

CREMONEZ, L. D.; DA SILVA, I. A. L. Tradução e Retradução de The Picture of Dorian Gray, de Oscar Wilde: um estudo de corpus com foco na apresentação do discurso. Domínios de Linguagem, v. 9, n. 2, jun/abr, p. 149-178, 2015 http://dx.doi.org/10.14393/dl18-v9n2a20159

DASTJERDI, H. V.; MOHAMMADI, A. Revisiting "retranslation hypothesis": a comparative analysis of stylistic features in the Persian retranslations of Pride and Prejudice. Open Journal of Modern Linguistics, v. 3, n. 3, p. 174-181, 2013. http://dx.doi.org/10.4236/ojml.2013.33024

DRAGSTED, B.; CARL, M. Towards a classification of translation styles based on eyetracking and keylogging data. Journal of Writing Research, v. 5, n. 1, p. 133-158, 2013. http://dx.doi.org/10.17239/jowr-2013.05.01.6

GAMBIER, Y. La retraduction, re tour et tour. Meta: Journal des Traducteurs, v. 39, p. $413-$ 417, 1994. http://dx.doi.org/10.7202/002799ar

GIANNOTTO, E. C. Uso de rastreamento ocular na avaliação da experiência do teleusuário de TV interativa. 290 f. 2009. Dissertação (Mestrado em Engenharia Elétrica) Escola Politécnica, Universidade de São Paulo, 2009.

HVELPLUND, K. T. Allocation of cognitive resources in translation: an eye-tracking and key-logging study. 260f Tese (Doutorado) - Copenhagen Business School, Copenhagen, 2011.

. Eye tracking and the process of dubbing translation. In DÍAZ-CINTAS, J.; NIKOLI, K. (Ed.). New pursuits in audiovisual translation. London: Multilingual Matters, 2015, p. 113.

JAKOBSEN, A. L.; JENSEN, K. T. H. Eye movement behavior. Copenhagen Studies in Language, v. 36, p. 103-124, 2008.

JUST, M. A.; CARPENTER, P. A theory of reading: from eye fixations to comprehension. Psychological Review, v. 85, p. 109-130, 1980. Disponível em: http://repository.cmu.edu/cgi/viewcontent.cgi?article=1731\&context=psychology. Último Acesso em: 13 maio 2014. 
MALTA, G. O processo cognitivo em tarefas de (re)tradução: um estudo baseado em rastreamento ocular, registro de teclado e mouse e protocolos verbais. $244 \mathrm{f}$. Tese (Doutorado em Linguística Aplicada) - Faculdade de Letras, Universidade Federal de Minas Gerais, Belo Horizonte, 2015.

MALTA, G.; RAEL, E. C. As retraduções de La casa de los espíritus: uma (re)visita à hipótese da retradução. Letras Raras, v. 4, n. 3, p. 63-74, 2015.

PALOPOSKI, O.; KOSKINEN, A. Reprocessing texts: the fine line between retranslating and revising. Across Languages and Cultures, v. 11, n. 1, p. 29-49, 2010. http://dx.doi.org/10.1556/Acr.11.2010.1.2

PAVLOVIĆ, N.; JENSEN, K. Eye-tracking translation directionality. In PYM, A.; PEREKRESTENKO, A. (Ed.). Translation Research Projects 2. Tarragona: Intercultural Studies Group, 2009. p. 93-109.

RAYNER, K. Eye movements in reading and information processing: 20 years of research. Psychological Bulletin, v. 124, p. 372-422, 1998. http://dx.doi.org/10.1037/00332909.124.3.372

RODRIGUES, C. A abordagem processual no estudo da tradução: uma meta-análise qualitativa. Cadernos de Tradução, v. 2, n. 10. In: ALVES, F. (Org.). O processo de tradução. Florianópolis: NUT-UFSC. 2002. p. 23-57.

SEKINO, K. Investigando processos de pós-edição e de tradução: uma análise cognitivopragmática da relação esforço-efeito no par linguístico japonês-português. $194 \mathrm{f}$. Tese (Doutorado em Linguística Aplicada) - Faculdade de Letras, Universidade Federal de Minas Gerais, Belo Horizonte, 2015

TAHIR-GÜRÇALAR, S. Retranslation. In: BAKER, M.; MALMKJAER, K. Routledge encyclopaedia of translation studies. 1. ed. Londres: Routledge, 1998. p. 125-127.

TIRKKONEN-CONDIT, S. Uncertainties in translation processes. In: TIRKKONENCONDIT, S.; JÄÄSKELÄINEN, R. Tapping and mapping the process of translation and interpreting. Amsterdã: John Benjamins, 2000. p. 123-142. http://dx.doi.org/10.1075/btl.37.13tir

VENUTI, L. The translator's invisibility. Nova Iorque: Routledge, 1995. http://dx.doi.org/10.4324/9780203360064

Escândalos da tradução: por uma ética da diferença. Trad. Laureano Pelegrin, Lucinéia Marcelino Villela, Marileide Dias Esqueda e Valéria Biondo. Bauru: EDUSC, 2002 